\title{
Analysis of 1060 Cases of Drug-Induced Acute Pancreatitis
}

\author{
Ágnes Meczker, ${ }^{1}$ Lilla Hanák, ${ }^{1}$ Andrea Párniczky, ${ }^{1,2}$ Andrea Szentesi, ${ }^{1,3}$ Bálint Erőss, ${ }^{1,4, \S}$ and \\ Péter Hegyi, ${ }^{1,3,4, \S}$ on behalf of the Hungarian Pancreatic Study Group
}

\begin{abstract}
${ }^{1}$ Institute for Translational Medicine, Medical School, University of Pécs, Pécs, Hungary; ${ }^{2}$ Department of Gastroenterology, Heim Pál National Pediatric Institute, Budapest, Hungary; ${ }^{3}$ Centre for Translational Medicine, Faculty of Medicine, University of Szeged, Szeged, Hungary; and ${ }^{4}$ Department of Gastroenterology, First Department of Medicine, Medical School, University of Pécs, Pécs, Hungary
\end{abstract}

Keywords: Drug-Induced Pancreatitis; Acute Pancreatitis; Mortality; Severity.

A cute pancreatitis (AP) has a mortality of approximately $3 \%{ }^{1}$ Its reported incidence is variable across countries (10 to $100 / 100,000$ inhabitants), and in the United States, AP is a significant cause of acute hospitalization for gastrointestinal disorders. ${ }^{2}$ Drug-induced AP (DIAP) is regarded as a rare and mild entity; yet, it is estimated to account for approximately $2 \%$ to $5 \%$ of AP episodes worldwide. ${ }^{3,4}$ Because DIAP has no unique features, rechallenge with the offending drug would be the only way to provide the most robust evidence to confirm the etiology. However, giving back the drug only for understanding the etiology of index AP is ethically unacceptable. ${ }^{3}$ Therefore, unsurprisingly, DIAP often remains a speculative diagnosis. A more detailed introduction of DIAP can be found in Supplementary Document 1.

We aimed to systematically search the medical literature, analyze the outcomes of all reported cases of DIAP, and compare them against a general cohort of AP.

\section{Methods}

We comprehensively searched the literature for reported cases of proven DIAP and extracted detailed data of each case on both first episodes and rechallenges. We compared DIAP to the large cohort of AP cases collected by the Hungarian Pancreatic Study Group. Details of the methods are in Supplementary Document 1.

\section{Results}

As a result of the comprehensive search and selection, we identified and analyzed 1060 eligible patients in 856 reports (details of the articles can be found in Supplementary Document 2).

\section{Epidemiology and Outcome Parameters of the First Episodes of Drug-Induced Acute Pancreatitis}

In a large proportion of the 1060 patients, antineoplastic (179 [16.89\%]), antibiotic (128 [12.08\%]), and anticonvulsant (103 [9.72\%]) drugs caused DIAP. A combination of drugs caused $78(7.36 \%)$ of the DIAP episodes (Figure 1A). In approximately half of the 1060 patients, drugs were given to manage the diseases of the gastrointestinal tract (214 [20.19\%]) or neurologic (158 [14.91\%]) or hematologic (155 [14.62\%]) conditions (Figure 1B). The 10 most common drugs resulting in AP are shown in Figure $1 C$.
Male patients comprised 536 of 1054 patients (50.85\%) affected by DIAP (Figure 1D). Interestingly, 228 of 1054 cases (21.63\%) were reported in children (aged $<18$ years), and cases were seen at a younger age than in AP of other common etiologies (Figure 1E). Among the 1060 patients, DIAP was severe in $213(20.09 \%)$, moderately severe in 118 (11.13\%), and mild in $729(68.77 \%)$, if the first episodes were analyzed (Figure $1 F$ ).

DIAP had a mortality of 90 of $1033(8.71 \%)$ for all severities (Figure $1 G$ ). There was a significant difference in the median (interquartile range [IQR]) length of hospitalization (LOH) between mild and moderately severe (7 days [IQR, 4-11.5 days] vs 16 days [IQR, 7-25 days], $P$.001) and between mild and severe DIAP (7 days [IQR, $4-11.5$ days] vs 18 days [IQR, 6.5-42 days], $P<.001)$. There was no difference in the LOH between moderately severe and severe DIAP (Figure $1 H$ ).

We found information on rechallenge in 960 of the 1060 patients in our analysis. Epidemiology (indication, sex, and age) and outcome parameters (severity, mortality, and LOH) of the rechallenge episodes of DIAP can be found in Figure $2 A-F$. The association of the drug categories and primary conditions with the severity and mortality rates of DIAP can be found in Supplementary Document 1 and Supplementary Tables 1 and 2.

\section{Effect of Dose on the Outcome of Drug-Induced Acute Pancreatitis After Rechallenge}

In 147 of 241 patients (70.00\%), no data were available on the dose for rechallenge. Rechallenge was performed in 49 of 241 patients $(20.33 \%)$ with the same dose as given in the first DIAP episode. The dose was decreased in 33 patients $(13.69 \%)$ and was increased in 12 (4.98\%) compared with the drug dose given in the first episode. If the same dose was given that provoked first episode, DIAP was severe in 2 of 41 patients (4.88\%), moderate in $3(7.32 \%)$, and mild in $36(87.80 \%)$. If decreased doses were given, we found no moderately severe cases. Rechallenge in 28 patients caused 1 (3.57\%) severe case and 27 (96.43\%) mild cases of DIAP (Figure $2 G$ ).

$\S$ Authors share co-senior authorship.

Abbreviations in this paper: AP, acute pancreatitis; DIAP, drug-induced acute pancreatitis; IQR, interquartile range; $L O H$, length of hospitalization.

Most current article

(C) 2020 by the AGA Institute. Published by Elsevier Inc. This is an open access article under the CC BY-NC-ND license (http://creativecommons. org/licenses/by-nc-nd/4.0/). 0016-5085

https://doi.org/10.1053/j.gastro.2020.07.016 
A DRUG GROUPS
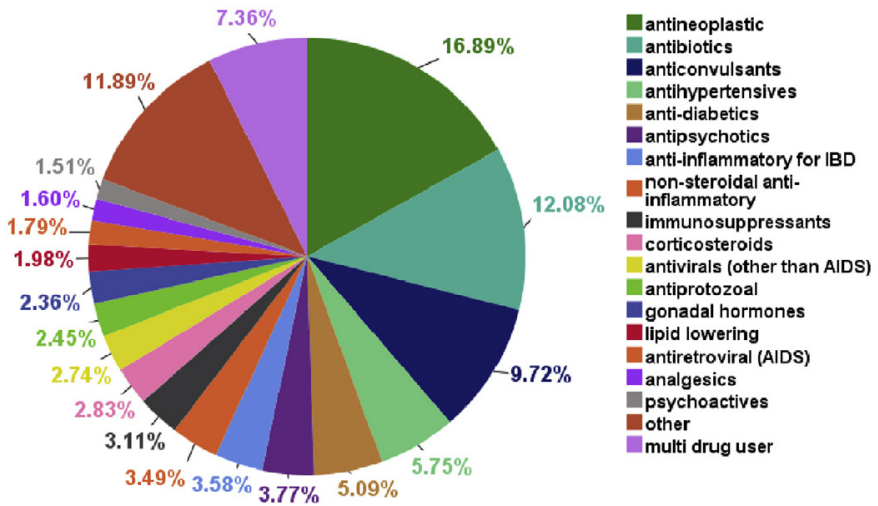

C SPECIFIC DRUGS

\begin{tabular}{|l|c|c|}
\hline \multicolumn{3}{|c|}{ TOP 10} \\
\hline Drug name & $\mathbf{n}$ & $\%$ \\
\hline Valproic acid & 81 & $7.64 \%$ \\
\hline L-asparaginase & 68 & $6.42 \%$ \\
\hline Mesalamine & 28 & $2.64 \%$ \\
\hline Azathioprine & 19 & $1.79 \%$ \\
\hline Ciprofloxacin & 17 & $1.60 \%$ \\
\hline Peg-Aspargase & 17 & $1.60 \%$ \\
\hline Prednisone & 17 & $1.60 \%$ \\
\hline Olanzapine & 16 & $1.51 \%$ \\
\hline Cannabis & 15 & $1.42 \%$ \\
\hline Isoniazid & 14 & $1.32 \%$ \\
\hline \multicolumn{3}{|c}{} \\
\hline
\end{tabular}

F SEVERITY

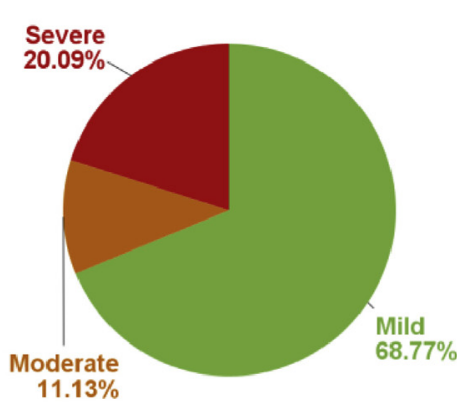

G
B

PRIMARY DISEASES

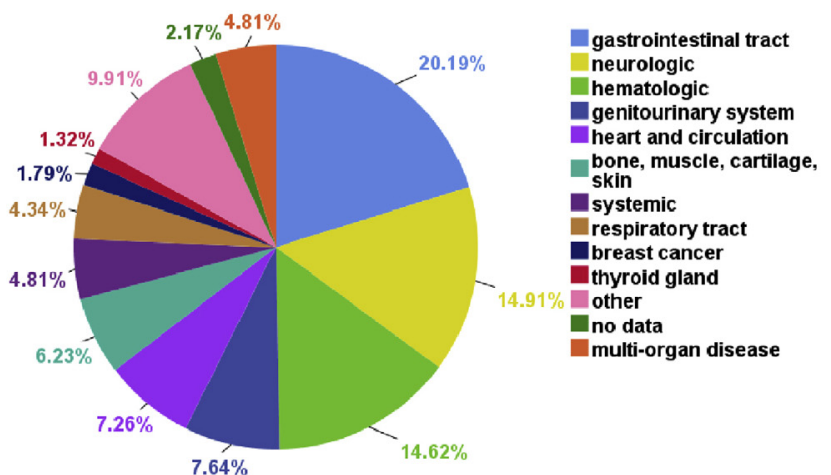

D GENDER
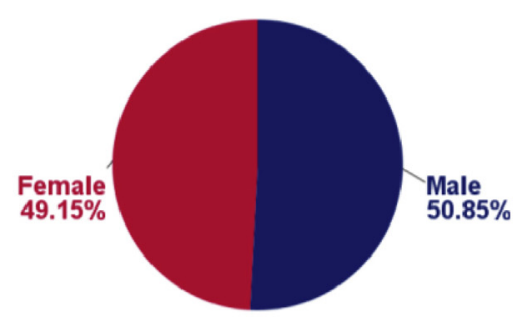

E

AGE

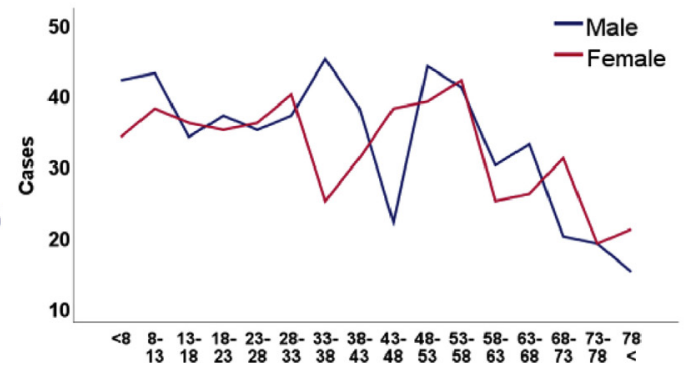

MORTALITY

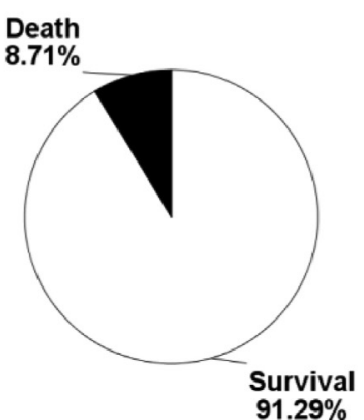

H

\section{LOH}

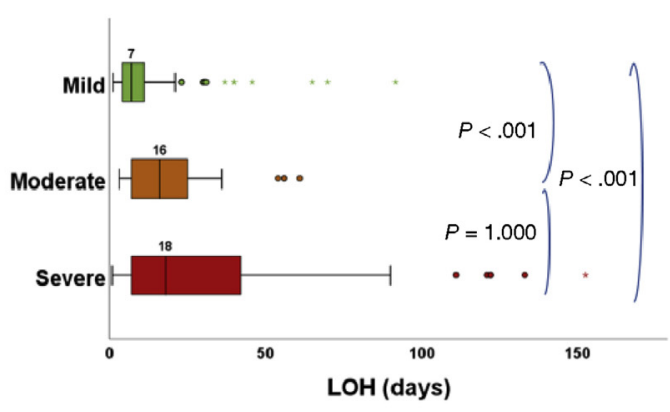

Figure 1. Epidemiology and outcome parameters of the first episodes of DIAP in 1060 patients. (A) Culprit drugs reported are subgrouped according to their mechanism of action. IBD, inflammatory bowel disease. (B) Primary diseases that were the reason for the drug intake are subgrouped according to the affected organ. $(C)$ The top 10 culprit drugs. $(D)$ Sex ratio of patients. (E) Age distribution of patients. $(F)$ Rate of the severities of cases. $(G)$ Mortality. $(H)$ LOH in the 3 different severities. The horizontal line in the middle of each box indicates the median; the top and bottom borders of the box mark the 75th and 25th percentiles, respectively; the whiskers mark the minimum and maximum of all of the data; and the circles indicate outliers.

\section{Analysis of Drug-Induced Acute Pancreatitis Versus the General Acute Pancreatitis Cohort}

The descriptive statistics of the general AP cohort are available in Supplementary Document 1 and Supplementary Figure 1. Our data showed that severity and mortality were increased in all DIAP cases compared with AP of other etiologies by $18.41 \%$ vs $5.63 \%(P<.001)$ and $7.30 \%$ vs $2.20 \%(P<$
.001), respectively. DIAP had the second highest mortality rate of all etiologies (8.49\%) (Supplementary Document 1 and Supplementary Figure 1).

\section{Discussion}

One of the most critical findings of our study is that compared with AP of other etiologies, reported patients 
$n=241$

A INDicAtion

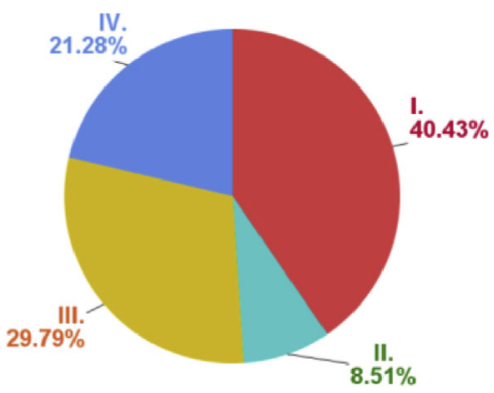

B GENDER

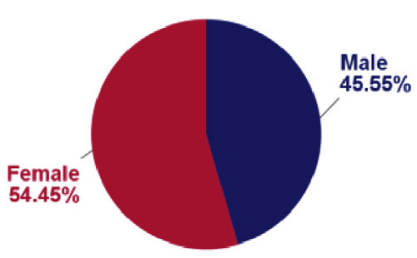

C
AGE

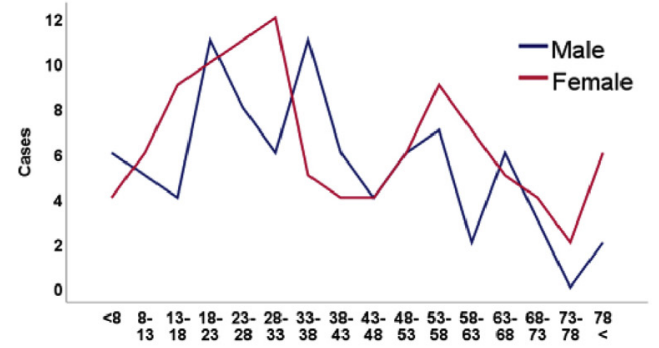

\section{SEVERITY E MORTALITY F LOH}
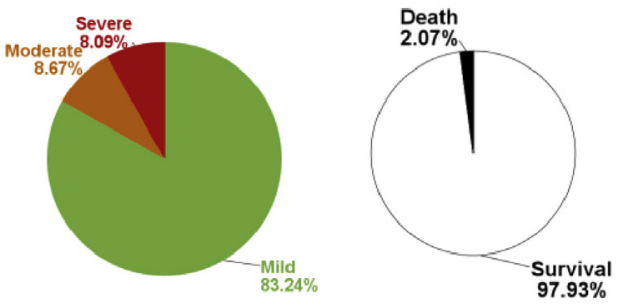

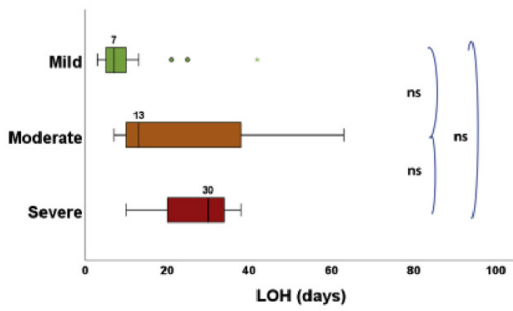

DOSES OF RECHALLENGE

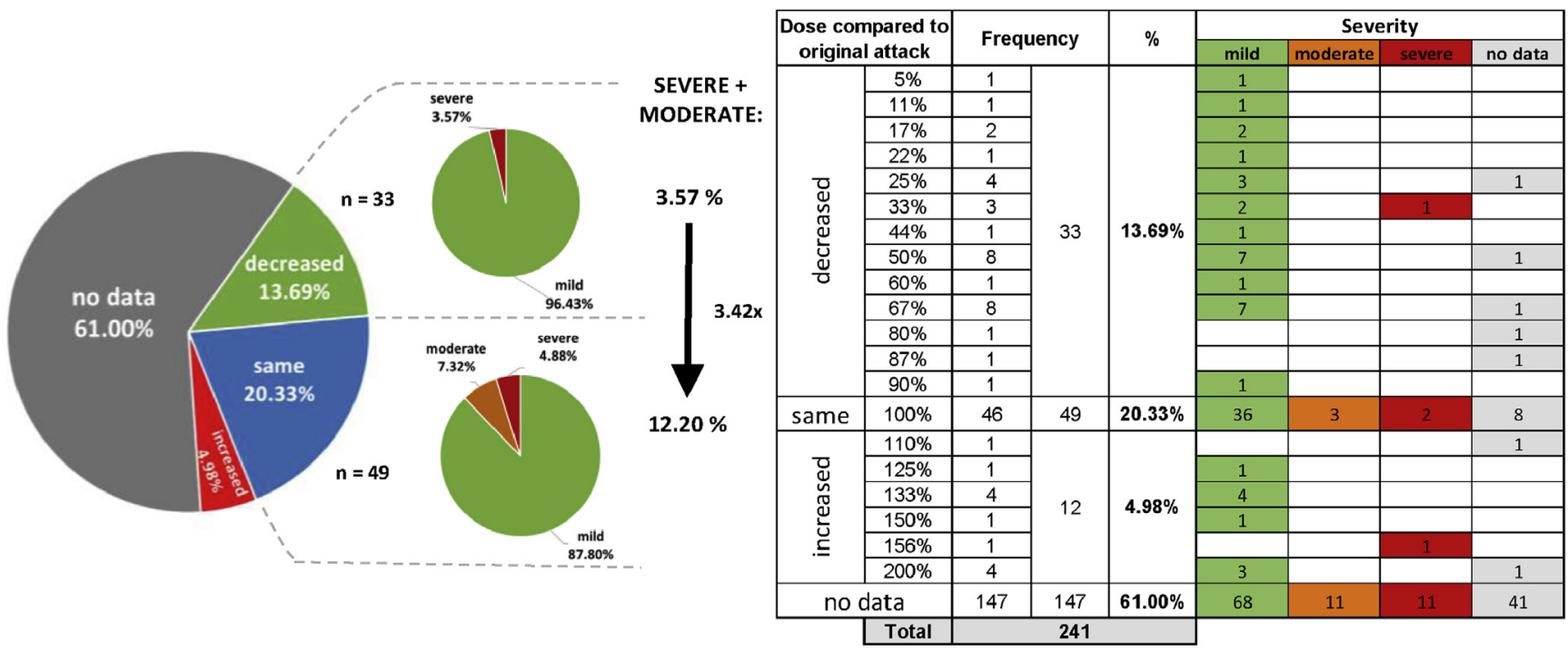

Figure 2. Characterization of rechallenge events in 241 patients. $(A)$ Reason for the rechallenge. $(B)$ Sex distribution of patients. $(C)$ Age distribution of patients. $(D)$ Severities of patients. $(E)$ Mortality. $(F)$ LOH in the 3 severities (ns, no significant difference). The horizontal line in the middle of each box indicates the median; the top and bottom borders of the box mark the 75th and 25th percentiles, respectively; the whiskers mark the minimum and maximum of all of the data; and the circles indicate outliers. (G) Correlation of drug doses and severity of DIAP in rechallenge. If the drug dose was the same during a rechallenge, the rate of moderately severe and moderate AP cases was 3.42 times more frequent compared with the cases where rechallenge was performed with decreased doses.

with DIAP have a more severe disease course. Most medications causing severe DIAP are given to treat significant preexisting pathologies and primary diseases such as cancers and autoimmune disorders. These patients will have a higher risk of organ failure. In some patients, organ failure is present at the introduction of the offending drug, before the
DIAP event. We hypothesize that this accounts for the increased proportion of moderately severe and severe cases of AP in the DIAP cohort.

A primary disease itself is a comorbidity and often has other comorbidities. We believe that the more severe the primary disease was, the higher doses of the offending 
drugs were used, leading to more severe courses of the DIAP patients. The offending drugs likely cause the DIAP in a dose-dependent way.

In our recent meta-analysis, older age led to a more severe disease course, ${ }^{5}$ and our recent cohort analysis proved that comorbidities are more critical in AP than age. ${ }^{6}$ These conclusions are in line with the findings of the present study and support our above-detailed hypothesis.

Besides the negative effect of comorbidities on the outcome of pancreatitis, culprit drugs have direct toxic effects on acinar cells as well. Asparaginase, for example, was shown to cause cellular necrosis. ${ }^{7}$ Importantly, here we report for the first time that when rechallenge was done with a decreased dose of the offending drug, it resulted in less severe outcomes. The main strength and limitations of this study are in Supplementary Document 1.

Here we conclude that reported cases of DIAP have worse outcomes than AP of other etiologies and seem to be dose-dependent. If rechallenge is necessary, we recommend that patients are closely monitored and receive a reduced drug dose. Evidence-based guidelines on DIAP and rechallenge should be developed.

\section{Supplementary Material}

Note: To access the supplementary material accompanying this article, visit the online version of Gastroenterology at www.gastrojournal.org, and at https://doi.org/10.1053/ j.gastro.2020.07.016.

\section{References}

1. Parniczky A, et al. PLoS One 2016;11:e0165309.

2. Peery AF, et al. Gastroenterology 2019;156:254-272. e11.
3. Nitsche C, et al. Curr Gastroenterol Rep 2012;14:131138.

4. Badalov N, et al. Clin Gastroenterol Hepatol 2007;5:648661.

5. Marta K, et al. Front Physiol 2019;10:328.

6. Szakacs Z, et al. Front Physiol 2018:9:1776.

7. Peng S, et al. Biol Sci 2016;37:1700.

Received February 20, 2020. Accepted July 13, 2020.

\section{Correspondence}

Address correspondence to: Péter Hegyi, MD, PhD, DSc, MAE, Institute for Translational Medicine, University of Pécs, Medical School, Szigeti út 12, 7624 Pécs, Hungary. e-mail: hegyi2009@gmail.com; fax: +36-7-253-6247.

\section{CRediT Authorship Contributions}

Ágnes Meczker MSC (Conceptualization: Equal; Data curation: Equal Investigation: Equal; Writing - original draft: Lead). Lilla Hanák MSC (Conceptualization: Equal; Data curation: Equal; Formal analysis: Lead; Writing - original draft: Equal). Andrea Párniczky, MD PhD (Formal analysis: Equal; Writing - review \& editing: Equal). Andrea Szentesi, PhD (Forma analysis: Equal; Writing - review \& editing: Equal). Bálint Erőss, MD, PhD (Conceptualization: Equal; Investigation: Equal; Writing - original draft: Lead). Péter Hegyi, MD, PhD, DSc, MAE (Conceptualization: Equal; Data curation: Equal; Writing - review \& editing: Lead).

\section{Appendix}

Hungarian Pancreatic Study Group: Erdősi D, Mikó A, Szakács Zs, Dobszai D, and Szapáry L: Institute for Translational Medicine, Medical School, Szentágothai Research Centre, University of Pécs, Pécs, Hungary; Bajor J, Mikó A, and Vincze A: Department of Gastroenterology, First Department of Medicine, Medical School, University of Pécs, Pécs, Hungary; Matuz M and Csupor D: Department of Clinical Pharmacy, Faculty of Pharmacy, University of Szeged, Hungary; Gábor Pethő: Department of Pharmacology and Pharmacotherapy, Medical School, University of Pécs, Pécs, Hungary; and Heim Pál: Children's Hospital, Budapest.

Conflicts of interest

The authors disclose no conflicts.

Funding

The research was supported by Project Grants (K131996 to P.H. and FK131864 to M.A.), an Economic Development and Innovation Operative Programme Grant (2.3.2-15-2016-00048 to P.H.), and a Human Resources Development Operational Programme Grant (EFOP-3.6.2-16-2017-00006 to P.H.) of the National Research, Development and Innovation Office. 


\section{Supplementary Material}

Drug-induced acute pancreatitis (DIAP) is regarded as a rare entity; yet, it is estimated to account for approximately $2 \%$ to $5 \%$ of acute pancreatitis (AP) episodes worldwide. ${ }^{1-5}$ However, estimates vary due to the challenging diagnosis and the difficulties of causality assessment. ${ }^{6-8}$ Because DIAP has no unique features that would help in distinguishing a case of DIAP from other etiologies, a rechallenge with the offending drug resulting a relapse of DIAP still means the most reliable evidence in confirming the etiology. ${ }^{3,9}$ In most cases, intentional rechallenge is considered unethical due to the potentially life-threatening complications of AP; therefore, DIAP remains a speculative diagnosis of exclusion.

The subject of past reviews is usually the categorization of the drugs based on their reported frequency of provoking DIAP $^{4,10}$ and the analysis of the strength of the causal relationship between the drug intake and the AP episode. ${ }^{11-}$

13 According to the literature, most cases of DIAP are mild, self-limited, and dose-independent, with a rapid resolution upon discontinuation of the offending drug. ${ }^{14}$ However, in our previous study on 5-aminosalicylic acid-induced DIAP, we found that DIAP might not be dose-independent, and we saw more moderately severe cases than expected. ${ }^{15}$

\section{Methods}

\section{Systematic Search}

We performed a systematic literature search according to Preferred Reporting Items for Systematic Reviews and Meta-Analyses (PRISMA) guideline. ${ }^{16}$ The review was registered on PROSPERO under the ID number CRD42017079196. The following PECO items were used: $\mathrm{P}=$ patients with AP; $\mathrm{E}=\mathrm{DIAP} ; \mathrm{C}=\mathrm{AP}$ caused by other etiologies; and $\mathrm{O}=$ severity, mortality, length of hospitalization (LOH), imaging alterations, symptoms, and time to resolution of AP. The search was performed in May 2019 on PubMed, EMBASE, and Cochrane Library with the search terms "acute pancreatitis" and "drug" and was limited to English-language and human studies (if applicable), regardless of the date of publication. Study selection was performed in parallel by 2 independent researchers. Studies that contained pooled statistical data of DIAP were excluded because they did not provide relevant data for our analysis.

\section{Inclusion and Exclusion Criteria}

Records that contained relevant data on patients with DIAP were eligible for our study irrespective of study design (case reports included as well). Cases reported as DIAP in which alcoholic or gallstone or different obvious etiology could be suspected were not included.

\section{Risk of Bias}

We developed an assessment tool for the reporting quality of the identified articles to exclude poorly reported cases, which would threaten data quality and the analysis.
We identified 3 categories of reporting quality, based on the reported symptoms and signs of AP (abdominal pain, pancreatic enzyme elevation, imaging changes) and their causality with the offending drug.

Strong evidence: The report contained data sufficient to reevaluate the event as DIAP.

Moderate evidence: The report described the event as DIAP, but data only partially confirmed it (could not be reevaluated as AP).

Weak evidence: The report described the event as DIAP, but there was no detailed data for reevaluation.

In our analysis, we included in the statistical analysis only cases with strong evidence levels.

\section{Definition of Acute Pancreatitis}

We reevaluated all events documented by the authors as AP. Each was considered as AP if it met the criteria detailed in the evidence-based guidelines for the management of AP. ${ }^{17,18}$

To assess severity, any organ failure reported by the authors was accepted, even if there were no supporting data. Persistent organ failure was defined that lasted longer than 48 hours or was described as persistent by the original authors themselves, transient organ failure was defined that resolved within 48 hours, or described as transient by the authors.

We accepted the pancreatic enzyme level elevation as higher than triple the upper limit of normal if (1) the exact enzyme level and the upper limit of normal were described and the enzyme level exceeded more than 3 times, (2) the precise extent of elevation compared with the upper limit of normal was provided and was more than 3-fold, and (3) the exact pancreatic enzyme levels were given without their references but were higher than $300 \mathrm{U} / \mathrm{L}$ in the case of amylase and $180 \mathrm{U} / \mathrm{L}$ in the case of lipase.

\section{Rechallenge}

We considered the result of rechallenge positive if a trial with the suspected offending drug resulted in the increase of the pancreatic enzyme levels, with or without abdominal pain, nausea, or vomiting.

\section{Severity of Drug-Induced Acute Pancreatitis}

To determine the severity of DIAP, we performed an evaluation using the data provided by the authors. We screened each case for the description of local and systemic complications and organ failure. If the detailed clinical data were available, the severity of DIAP was determined by the modified Atlanta criteria, irrespective of the classification by the original authors. If the lack of clinical data did not allow us to determine the severity of DIAP, we used the severity grade reported by the authors.

\section{Primary Disease and Drug Categorization}

The offending drugs were given to manage specific disorders. We defined these as the primary diseases. 


\section{Acute Pancreatitis Cohort}

For the comparative statistical analysis of the DIAP cases to analyze them against AP of other etiologies, we used the detailed clinical data of the AP cohort of the Hungarian Pancreatic Study Group, as described in our previous studies $^{19-22}$

\section{Interpretation of Data}

We used descriptive statistical tools to characterize the population, and relative frequency and median and interquartile range were calculated. To analyze the differences between the severity groups for the $\mathrm{LOH}$ and the time that the enzyme level and symptoms normalized, we applied the Kruskal-Wallis test with the Mann-Whitney $U$ test as a post hoc test. Differences between drug and disease categories and differences for DIAP against other etiologies of AP were examined using the $\chi^{2}$ test. We regarded a $P$ value of $<.05$ as statistically significant. The available-case analysis was used for missing data. Statistical analyses were performed using IBM-SPSS for Windows 25 software (IBM Corp, Armonk, NY).

\section{Additional Results}

\section{Outcomes of Drug-Induced Acute Pancreatitis Compared With Acute Pancreatitis With Other Etiologies}

We compared the severity and mortality rates of DIAP to AP caused by the more common etiologies like biliary diseases, idiopathic etiology, alcohol consumption, lipid metabolism disorder, status after endoscopic retrograde cholangiopancreatography, and the combinations of these. The detailed descriptive statistics of the AP cohort are shown in Supplementary Figure 1.

We found that DIAP showed the most severe episodes if only the first episodes were analyzed (213 of 1060 [20.09\%]; Supplementary Figure 1). If the severe cases of first and rechallenged events were pooled, the rate of severe cases was slightly but not significantly lower (227 of 1301 [18.41\%]). Significant differences were seen between the rate of severe and mortality rates of DIAP cases if the first episodes are compared with rechallenges, at $20.09 \%$ vs 8.09\% $(P<.001)$ and $8.49 \%$ vs $2.07 \%(P<.001)$, respectively. Severity and mortality were increased in all DIAP compared with cases with all other etiologies, at $18.41 \%$ vs $5.63 \%(P<.001)$ and $7.30 \%$ vs. $2.20 \%(P<.001)$, respectively. DIAP had the second highest mortality rate of all etiologies (8.49\%). Only AP of combined alcoholic and biliary etiology had a similarly high mortality rate $(8.7 \%$; Supplementary Figure 1).

\section{The Association of the Drug Categories With the Severity and Mortality Rates of Drug-Induced Acute Pancreatitis}

Antiprotozoal drugs, corticosteroids, and antiretrovirals were responsible for the most severe cases of DIAP in 12 of 26 patients (46.15\%), 13 of 30 (43.33\%), and 7 of 19 (36.84\%), respectively. Corticosteroids, antiprotozoal drugs, and antiretrovirals had the highest mortality rates, at 12 of 30 patients (40.00\%), 8 of 26 (30.77\%), and 5 of $19(26.32 \%)$, respectively. Patients who were taking antiprotozoals (46.15\%; $P<.01)$, corticosteroids (43.33\%; $P<.01$ ), antihypertensives (29.51\%; $P<.05)$, and antineoplastics $(24.58 \% ; P$ $<.001$ ) had a higher chance of severe disease than patients taking other drugs. In contrast to this, patients taking antiinflammatory drugs for inflammatory bowel disease (10.53\%; $P<.5)$, antibiotics (7.03\%; $P<.001)$, or other drugs than the specified ones $(14.29 \% ; P<.05)$ had a lower chance of a severe episode than patients taking other drugs. Patients taking anticonvulsants had a significantly higher chance of moderately severe DIAP $(24.27 \%$; $P<.001)$ than patients taking other drugs. Patients taking corticosteroids $(40.00 \% ; P$ $<.001$ ), antiprotozoals (30.77\%; $P<.01$ ), antiretrovirals (26.32\%; $P<.05)$, antihypertensives (21.31\%; $P<.01)$, and patients on multiple medications $(15.58 \% ; P<.05)$ had a higher chance of mortality than those taking other drugs. However, patients on antibiotics had a smaller chance of mortality than patients taking other drugs $(3.15 \%$; $P<.05$; Supplementary Table 1).

\section{The Association of the Primary Conditions With the Severity and Mortality Rates of Drug-Induced Acute Pancreatitis}

The severity of DIAP was analyzed for subgroups of primary diseases, which showed that patients with breast cancer, hematologic conditions, and cardiovascular failure had the highest rates of severe DIAP, at 6 of 19 (31.58\%), 45 of 155 (29.03\%), and 20 of 77 (25.97\%), respectively. Underlying gastrointestinal tract disease had a lower chance of severe DIAP $12.15 \% ; P$ $<$.001) than other diseases. Hematologic disorders had a higher rate of a severe DIAP episode $(29.03 \% ; P<.001)$ than in other conditions. Neurologic conditions had a significantly higher chance for a moderately severe DIAP $(20.89 \% ; P<.001)$ than in other diseases. Mortality was lower among patients with gastrointestinal tract disease $(3.38 \% ; P<.01)$ and higher in multiple diseases (19.61\%; $P<.05)$, heart and circulatory diseases (19.48\%; $P<.001)$, and systemic diseases $(17.65 \% ; P$ $<.05$ ) than in other diseases (Supplementary Table 2).

\section{Strengths}

To our knowledge, this is the only study that comprehensively searched and identified all DIAP cases in the literature. We followed a rigorous methodology, including data extraction and quality analysis of each individual article, to generate an extensive database of reported cases of DIAP. We used this broad database to analyze the natural history of DIAP. Our systematic and comprehensive search identified and resulted in a very detailed data of 1060 cases of DIAP. To date, this is the largest and most comprehensive analysis of all reported cases of DIAP. Because we collected data on the first episodes of DIAP and on rechallenges, we could compare the 2 entities.

\section{Limitations}

This study is based on data extracted from case reports and case series, which introduces all of the limitations of 
the genre, most importantly, recall and publication bias. ${ }^{23}$ The publication bias was increased by the English language filter, which we had to use due to a large number of records identified by the preliminary search. Case reports and series publications, which are almost always written in retrospect, may contain insufficient data, which is a concern.

The latency period between drug exposure and the start of the pancreatitis episode was not defined, and this is a

\section{References}

1. Eland IA, Van Puijenbroek EP, Sturkenboom MJCM, Wilson JHP, Stricker BHC. Drug-associated acute pancreatitis: twenty-one years of spontaneous reporting in the Netherlands. Am J Gastroenterol 1999;94:24172422.

2. Trivedi CD, Pitchumoni CS. Drug-induced pancreatitis: an update. J Clin Gastroenterol 2005;39:709-716.

3. Nitsche C, Maertin S, Scheiber J, Ritter CA, Lerch MM, Mayerle J. Drug-induced pancreatitis. Curr Gastroenterol Rep 2012;14:131-138.

4. Balani AR, Grendell JH. Drug-induced pancreatitis: incidence, management and prevention. Drug Saf 2008; 31:823-837.

5. Vinklerová I, Procházka M, Procházka V, Urbánek K. Incidence, severity, and etiology of drug-induced acute pancreatitis. Dig Dis Sci 2010;55:2977-2981.

6. Badalov N, Baradarian R, Iswara K, Li J, Steinberg W, Tenner S. Drug-induced acute pancreatitis: an evidencebased review. Clin Gastroenterol Hepatol 2007;5:648661; quiz 664.

7. Naranjo CA, Busto U, Sellers EM, et al. A method for estimating the probability of adverse drug reactions. Clin Pharmacol Ther 1981;30:239-245.

8. Karch FE, Lasagna L. Adverse drug reactions. A critical review. JAMA 1975;234:1236-1241.

9. Jones MR, Hall OM, Kaye AM, Kaye AD. Drug-induced acute pancreatitis: a review. Ochsner J 2015;15:45-51.

10. Spanier BW, Tuynman HA, van der Hulst RW, Dijkgraaf MG, Bruno MJ. Acute pancreatitis and concomitant use of pancreatitis-associated drugs. Am J Gastroenterol 2011;106:2183-2188.

11. Mallory A, Kern F Jr. Drug-induced pancreatitis: a critical review. Gastroenterology 1980;78:813-820.

12. McArthur KE. Review article: drug-induced pancreatitis. Aliment Pharmacol Ther 1996;10:23-38. limitation of our study. Owing to the lack of data, Naranjo's score could not be used, ${ }^{23}$ which is another significant limitation of the interpretation of the results.

The definition of DIAP after rechallenge needs careful consideration. The fluctuation of pancreatic enzyme levels and incomplete resolution of morphologic changes after an episode of acute pancreatitis make the clinical assessment of recurrent pancreatitis difficult, following the rechallenge with the suspected drug.

13. Wilmink T, Frick TW. Drug-induced pancreatitis. Drug Saf 1996;14:406-423.

14. Lankisch PG, Dröge M, Gottesleben F. Drug induced acute pancreatitis: incidence and severity. Gut 1995; $37: 565-567$.

15. Meczker A, Miko A, Hegyi P. 5-ASA induces mild acute pancreatitis. Case report and review of the literature. J Gastrointestin Liver Dis 2018;27:189-194.

16. Moher D, Shamseer L, Clarke M, et al. Preferred Reporting Items for Systematic Review and Metaanalysis protocols (PRISMA-P) 2015 statement. Syst Rev 2015;4:1.

17. Working Group IAP/APA Acute Pancreatitis Guidelines. IAP/APA evidence-based guidelines for the management of acute pancreatitis. Pancreatology 2013;13(Suppl 2):e1-e15.

18. Hritz I, Czakó L, Dubravcsik Z, et al. Acute pancreatitis. Evidence-based practice guidelines, prepared by the Hungarian Pancreatic Study Group. Orv Hetil 2015; 156:244-261; [in Hungarian].

19. Szakacs Z, Gede N, Pecsi D, et al. Aging and comorbidities in acute pancreatitis II: a cohort-analysis of 1203 prospectively collected cases. Front Physiol 2018;9:1776.

20. Farkas N, Hanák L, Mikó A, et al. A multicenter, international cohort analysis of 1435 cases to support clinical trial design in acute pancreatitis. Front Physiol 2019;10:1092.

21. Parniczky A, Lantos T, Toth EM, et al. Antibiotic therapy in acute pancreatitis: from global overuse to evidence based recommendations. Pancreatology 2019;19:488-499.

22. Szentesi A, Párniczky A, Vincze Á, et al. Multiple hits in acute pancreatitis: components of metabolic syndrome synergize each other's deteriorating effects. Front Physiol 2019;10:1202.

23. Nissen T, Wynn R. The clinical case report: a review of its merits and limitations. BMC Res Notes 2014;7:264. 


\section{Supplementary Document 2}

Preferred Reporting Items for Systematic Review and Meta-analysis Details of studies 
Supplementary Table 1.The Association of the Drug Categories With the Severity and Mortality Rates of Drug-Induced Acute Pancreatitis

\begin{tabular}{|c|c|c|c|c|c|c|c|c|c|c|c|c|c|c|c|}
\hline \multirow[b]{3}{*}{ Drug } & & \multicolumn{6}{|c|}{ Ranking based on severity (class of drugs) } & & & & & & & & \multirow[b]{3}{*}{ Severity } \\
\hline & & \multirow[b]{2}{*}{ Frequency } & \multicolumn{3}{|c|}{ Severity } & \multicolumn{2}{|c|}{ Mortality } & \multicolumn{2}{|c|}{ Sex } & \multicolumn{5}{|c|}{ Age, $y$} & \\
\hline & & & Severe & Moderate & Mild & Yes & No & Male & Female & $<18$ & $18-30$ & $30-50$ & $50-70$ & $\geq 70$ & \\
\hline \multirow[t]{2}{*}{ Antiprotozoals } & $\mathrm{n}$ & 26 & 12 & $\begin{array}{c}2 \\
7.69\end{array}$ & 12 & 8 & 18 & 18 & 8 & 0 & 13 & 13 & 0 & 0 & $46.15 \%$ \\
\hline & $\%$ & 2.45 & $46.15^{a}$ & 7.69 & 46.15 & $30.77^{a}$ & 69.23 & 69.23 & 30.77 & 0.00 & 50.00 & 50.00 & 0.00 & 0.00 & \\
\hline \multirow[t]{2}{*}{ Corticosteroids } & $n$ & 30 & 13 & 3 & 14 & 12 & 18 & 18 & 12 & 11 & 4 & 6 & 8 & 1 & \\
\hline & $\%$ & 2.83 & $43.33^{a}$ & 10.00 & 46.67 & $40.00^{b}$ & 60.00 & 60.00 & 40.00 & 36.67 & 13.30 & 20.00 & 26.67 & 3.33 & \\
\hline \multirow[t]{2}{*}{ Antiretrovirals (AIDS) } & $\mathrm{n}$ & 19 & 7 & 3 & 9 & 5 & 14 & 11 & 8 & 3 & 3 & 10 & 3 & 0 & \\
\hline & $\%$ & 1.79 & 36.84 & 15.79 & 47.37 & $26.32^{c}$ & 73.68 & 57.89 & 42.11 & 15.79 & 15.79 & 52.63 & 15.79 & 0.00 & \\
\hline \multirow[t]{2}{*}{ Antipsychotics } & $n$ & 40 & 12 & 6 & 22 & 3 & 37 & 26 & 14 & 2 & 7 & 21 & 6 & 3 & \\
\hline & $\%$ & 3.77 & 30.00 & 15.00 & 55.00 & 7.50 & 92.50 & 65.00 & 35.00 & 5.13 & 17.95 & 53.85 & 15.38 & 7.69 & \\
\hline \multirow[t]{2}{*}{ Antihypertensives } & $\mathrm{n}$ & 61 & 18 & 1 & 42 & 13 & 48 & 34 & 27 & 0 & 7 & 13 & 33 & 8 & \\
\hline & $\%$ & 5.75 & $29.51^{\circ}$ & 1.64 & 68.85 & $21.31^{a}$ & 78.69 & 55.74 & 44.26 & 0.00 & 11.48 & 21.31 & 54.10 & 13.11 & \\
\hline \multirow[t]{2}{*}{ Analgesics (non-NSAIDs) } & $n$ & 17 & 5 & 0 & 12 & 1 & 16 & 3 & 14 & 2 & 4 & 6 & 4 & 1 & \\
\hline & $\%$ & 1.60 & 29.41 & 0.00 & 70.59 & 5.88 & 94.12 & 17.65 & 82.35 & 11.76 & 23.53 & 35.29 & 23.53 & 5.88 & \\
\hline \multirow[t]{2}{*}{ Gonadal hormones } & $\mathrm{n}$ & 25 & 7 & 3 & 15 & 1 & 24 & 8 & 17 & 0 & 8 & 16 & 1 & 0 & \\
\hline & $\%$ & 2.36 & 28.00 & 12.00 & 60.00 & 4.00 & 96.00 & 32.00 & 68.00 & 0.00 & 32.00 & 64.00 & 4.00 & 0.00 & \\
\hline \multirow[t]{2}{*}{ Antidiabetics } & $n$ & 54 & 15 & 6 & 33 & 3 & 51 & 24 & 30 & 0 & 2 & 14 & 28 & 10 & \\
\hline & $\%$ & 5.09 & 27.78 & 11.11 & 61.11 & 5.56 & 94.44 & 44.44 & 55.56 & 0.00 & 3.70 & 25.93 & 51.85 & 18.52 & \\
\hline \multirow[t]{2}{*}{ Antineoplastics } & $\mathrm{n}$ & 179 & 44 & 35 & 100 & 8 & 147 & 83 & 96 & 86 & 18 & 30 & 37 & 8 & \\
\hline & $\%$ & 16.89 & 24.58 & 19.55 & 55.87 & 5.16 & 94.84 & 46.37 & 53.63 & 48.04 & 10.06 & 16.76 & 20.67 & 4.47 & \\
\hline \multirow[t]{2}{*}{ Anticonvulsants } & $n$ & 103 & 19 & 25 & 59 & 9 & 94 & 61 & 39 & 62 & 15 & 12 & 7 & 5 & \\
\hline & $\%$ & 9.72 & 18.45 & 24.27 & 57.28 & 8.74 & 91.26 & 61.00 & 39.00 & 61.39 & 14.85 & 11.88 & 6.93 & 4.95 & \\
\hline \multirow[t]{2}{*}{ Immunosuppressants } & $\mathrm{n}$ & 33 & 4 & 2 & 27 & 0 & 32 & 19 & 13 & 10 & 8 & 6 & 8 & 1 & \\
\hline & $\%$ & 3.11 & 12.12 & 6.06 & 81.82 & 0.00 & 100.00 & 59.38 & 40.63 & 30.30 & 24.24 & 18.18 & 24.24 & 3.03 & \\
\hline \multirow[t]{2}{*}{ NSAIDs } & $n$ & 37 & 4 & 1 & 32 & 0 & 37 & 15 & 22 & 6 & 5 & 7 & 10 & 9 & \\
\hline & $\%$ & 3.49 & 10.81 & 2.07 & 86.49 & 0.00 & 100.00 & 40.54 & 59.46 & 16.22 & 13.51 & 18.92 & 27.03 & 24.32 & \\
\hline Anti-inflammatory drugs for IBD & $\mathrm{n}$ & 38 & 4 & 1 & 33 & 2 & 36 & 16 & 22 & 9 & 12 & 16 & 1 & 0 & \\
\hline & $\%$ & 3.58 & $10.53^{c}$ & 2.63 & 86.84 & 5.26 & 94.74 & 42.11 & 57.89 & 23.68 & 31.58 & 42.11 & 2.63 & 0.00 & \\
\hline Antivirals (other than AIDS) & $n$ & 29 & 3 & 2 & 24 & 1 & 28 & 17 & 12 & 1 & 3 & 12 & 11 & 2 & \\
\hline & $\%$ & 2.74 & 10.34 & 6.90 & 82.76 & 3.45 & 96.55 & 58.62 & 41.38 & 3.45 & 10.34 & 41.38 & 37.93 & 6.90 & \\
\hline Lipid-lowering drugs & $n$ & 21 & 2 & 2 & 17 & 2 & 19 & 8 & 13 & 0 & 1 & 3 & 11 & 6 & \\
\hline & $\%$ & 1.98 & 9.52 & 9.52 & 80.95 & 9.52 & 90.48 & 38.10 & 61.90 & 0.00 & 4.78 & 14.29 & 52.38 & 28.57 & \\
\hline Antibiotics & $\mathrm{n}$ & 128 & 9 & 9 & 110 & 4 & 123 & 58 & 69 & 12 & 28 & 34 & 30 & 22 & \\
\hline & $\%$ & 12.08 & $7.03^{\circ}$ & 7.03 & 85.94 & $3.15^{c}$ & 96.85 & 45.67 & 54.33 & 9.52 & 22.22 & 26.98 & 23.81 & 17.46 & \\
\hline Psychoactives & $\mathrm{n}$ & 16 & 1 & 2 & 13 & 1 & 15 & 15 & 1 & 1 & 10 & 4 & 0 & 1 & $6.25 \%$ \\
\hline & $\%$ & 1.51 & 6.25 & 12.50 & 81.25 & 6.25 & 93.75 & 93.75 & 6.25 & 6.25 & 62.50 & 25.00 & 0.00 & 6.25 & \\
\hline Other & $n$ & 126 & 18 & 8 & 100 & 5 & 121 & 61 & 64 & 18 & 14 & 37 & 36 & 20 & \\
\hline & $\%$ & 11.89 & $14.29^{\circ}$ & 6.35 & 79.37 & 3.97 & 96.03 & 48.80 & 51.20 & 14.40 & 11.20 & 29.60 & 28.80 & 16.00 & \\
\hline On multiple medications & $\mathrm{n}$ & 78 & 16 & 7 & 55 & 12 & 65 & 41 & 37 & 5 & 13 & 19 & 32 & 9 & \\
\hline & $\%$ & 7.36 & 20.51 & 8.97 & 70.51 & $15.58^{c}$ & 84.42 & 52.56 & 47.44 & 6.41 & 16.67 & 24.36 & 41.03 & 11.54 & \\
\hline Total & $n$ & 1060 & 213 & 118 & 729 & 90 & 943 & 536 & 518 & 228 & 175 & 279 & 266 & 106 & \\
\hline & $\%$ & 100.00 & 20.09 & 11.13 & 68.77 & 8.71 & 91.29 & 50.85 & 49.15 & 21.63 & 16.60 & 26.47 & 25.24 & 10.06 & \\
\hline
\end{tabular}

IBD, inflammatory bowel disease; NSAID, non-steroidal anti-inflammatory drug.

${ }^{a} P<.01$.

${ }^{b} P<.001$.

${ }^{c} P<.05$. 
Supplementary Table 2. The Association of the Primary Conditions With the Severity and Mortality Rates of Drug-Induced Acute Pancreatitis

\begin{tabular}{|c|c|c|c|c|c|c|c|c|c|c|c|c|c|c|c|c|}
\hline \multicolumn{17}{|c|}{ Ranking based on severity (organ systems affected by the primary diseases) } \\
\hline \multirow[b]{2}{*}{ Primary condition } & & \multirow[b]{2}{*}{ Frequency } & \multicolumn{3}{|c|}{ Severity } & \multicolumn{2}{|c|}{ Mortality } & \multicolumn{2}{|c|}{ Sex } & \multicolumn{5}{|c|}{ Age, $y$} & \multirow{2}{*}{\multicolumn{2}{|c|}{ Severity }} \\
\hline & & & Severe & Moderate & Mild & Yes & No & Male & Female & $<18$ & $18-30$ & $30-50$ & $50-70$ & $\geq 70$ & & \\
\hline \multirow[t]{2}{*}{ Breast cancer } & $\mathrm{n}$ & 19 & 6 & 3 & 10 & 0 & 19 & 0 & 19 & 0 & 0 & 13 & 6 & 0 & & $31.58 \%$ \\
\hline & $\%$ & 1.79 & 31.58 & 15.79 & 52.63 & 0.00 & 100.00 & 0.00 & 100.00 & 0.00 & 0.00 & 68.42 & 31.58 & 0.00 & & \\
\hline \multirow[t]{2}{*}{ Hematologic } & $\mathrm{n}$ & 155 & 45 & 29 & 81 & 15 & 122 & 84 & 71 & 90 & 23 & 22 & 17 & 3 & & \\
\hline & $\%$ & 14.62 & $29.03^{a}$ & 18.71 & 52.26 & 10.95 & 89.05 & 54.19 & 45.81 & 58.06 & 14.84 & 14.19 & 10.97 & 1.94 & & \\
\hline \multirow[t]{2}{*}{ Heart and circulation } & $\mathrm{n}$ & 77 & 20 & 3 & 54 & 15 & 62 & 39 & 38 & 1 & 9 & 14 & 38 & 15 & & \\
\hline & $\%$ & 7.26 & 25.97 & 3.90 & 70.13 & $19.48^{a}$ & 80.52 & 50.65 & 49.35 & 1.30 & 11.69 & 18.18 & 49.35 & 19.48 & & \\
\hline \multirow[t]{2}{*}{ Respiratory tract } & $\mathrm{n}$ & 46 & 10 & 3 & 33 & 5 & 40 & 23 & 23 & 3 & 6 & 14 & 17 & 6 & & \\
\hline & $\%$ & 4.34 & 21.74 & 6.52 & 71.74 & 11.11 & 88.89 & 50.00 & 50.00 & 6.52 & 13.04 & 30.43 & 36.96 & 13.04 & & \\
\hline \multirow[t]{2}{*}{ Systemic } & $\mathrm{n}$ & 51 & 11 & 3 & 37 & 9 & 42 & 26 & 25 & 10 & 8 & 13 & 14 & 6 & & \\
\hline & $\%$ & 4.81 & 21.57 & 5.88 & 72.55 & $17.65^{b}$ & 82.35 & 50.98 & 49.02 & 19.61 & 15.69 & 25.49 & 27.45 & 11.76 & & \\
\hline \multirow[t]{2}{*}{ Neurologic } & $\mathrm{n}$ & 158 & 31 & 33 & 94 & 10 & 148 & 88 & 69 & 62 & 28 & 38 & 20 & 9 & & \\
\hline & $\%$ & 14.91 & $19.62^{a}$ & 20.89 & 59.49 & 6.33 & 93.67 & 56.05 & 43.95 & 39.49 & 17.83 & 24.20 & 12.74 & 5.73 & & \\
\hline \multirow[t]{2}{*}{ Bone, muscle, cartilage, skin } & $\mathrm{n}$ & 66 & 11 & 9 & 46 & 3 & 62 & 35 & 30 & 11 & 5 & 12 & 20 & 16 & & \\
\hline & $\%$ & 6.23 & 16.67 & 13.64 & 69.70 & 4.62 & 95.38 & 53.85 & 46.15 & 17.19 & 7.81 & 18.75 & 31.25 & 25.00 & & \\
\hline \multirow[t]{2}{*}{ Genitourinary system } & $\mathrm{n}$ & 81 & 13 & 6 & 62 & 7 & 74 & 26 & 54 & 6 & 19 & 33 & 17 & 6 & & \\
\hline & $\%$ & 7.64 & 16.05 & 7.41 & 76.54 & 8.64 & 91.36 & 32.50 & 67.50 & 7.41 & 23.46 & 40.74 & 20.99 & 7.41 & & \\
\hline \multirow[t]{2}{*}{ Gastrointestinal tract } & $\mathrm{n}$ & 214 & 26 & 16 & 172 & 7 & 200 & 112 & 101 & 21 & 31 & 52 & 79 & 30 & & \\
\hline & $\%$ & 20.19 & $12.15^{a}$ & 7.48 & 80.37 & $3.38^{c}$ & 96.62 & 52.58 & 47.42 & 9.86 & 14.55 & 24.41 & 37.09 & 14.08 & & \\
\hline \multirow[t]{2}{*}{ Thyroid gland } & $\mathrm{n}$ & 14 & 1 & 1 & 12 & 0 & 14 & 2 & 12 & 2 & 0 & 4 & 5 & 3 & & $7.14 \%$ \\
\hline & $\%$ & 1.32 & 7.14 & 7.14 & 85.71 & 0.00 & 100.00 & 14.29 & 85.71 & 14.29 & 0.00 & 28.57 & 35.17 & 21.43 & & \\
\hline \multirow[t]{2}{*}{ Other } & $\mathrm{n}$ & 105 & 21 & 8 & 76 & 7 & 98 & 59 & 44 & 14 & 27 & 35 & 18 & 9 & & \\
\hline & $\%$ & 9.91 & 20.00 & 7.62 & 72.38 & 6.67 & 93.33 & 57.28 & 42.72 & 13.59 & 26.21 & 33.98 & 17.48 & 8.74 & & \\
\hline \multirow[t]{2}{*}{ Multiple diseases } & $\mathrm{n}$ & 51 & 16 & 4 & 31 & 10 & 41 & 31 & 20 & 7 & 14 & 21 & 7 & 2 & & \\
\hline & $\%$ & 4.81 & 31.37 & 7.84 & 60.78 & $19.61^{b}$ & 80.39 & 60.78 & 39.22 & 13.73 & 27.45 & 41.18 & 13.73 & 3.92 & & \\
\hline \multirow[t]{2}{*}{ No data } & $\mathrm{n}$ & 23 & 2 & 0 & 21 & 2 & 21 & 11 & 12 & 1 & 5 & 8 & 8 & 1 & & \\
\hline & $\%$ & 2.17 & 8.70 & 0.00 & 91.30 & 8.70 & 91.30 & 47.83 & 52.17 & 4.35 & 21.74 & 34.78 & 34.78 & 4.35 & & \\
\hline
\end{tabular}


Ranking based on severity (organ systems affected by the primary diseases)

\begin{tabular}{|c|c|c|c|c|c|c|c|c|c|c|c|c|c|c|c|}
\hline \multirow[b]{2}{*}{ Primary condition } & & \multirow[b]{2}{*}{ Frequency } & \multicolumn{3}{|c|}{ Severity } & \multicolumn{2}{|c|}{ Mortality } & \multicolumn{2}{|c|}{ Sex } & \multicolumn{5}{|c|}{ Age, $y$} & \multirow[b]{2}{*}{ Severity } \\
\hline & & & Severe & Moderate & Mild & Yes & No & Male & Female & $<18$ & $18-30$ & $30-50$ & $50-70$ & $\geq 70$ & \\
\hline \multirow[t]{2}{*}{ Total } & $\mathrm{n}$ & 1060 & 213 & 118 & 729 & 90 & 943 & 536 & 518 & 228 & 175 & 279 & 266 & 106 & \\
\hline & $\%$ & 100 & 20.09 & 11.13 & 68.77 & 8.71 & 91.29 & 50.85 & 49.15 & 21.63 & 16.60 & 26.47 & 25.24 & 10.06 & \\
\hline
\end{tabular}

${ }^{a} P<.001$.

${ }^{b} P<.05$.

${ }^{c} P<.01$. 


\section{GENERAL COHORT OF AP}

$$
\mathbf{n}=\mathbf{1 2 2 5}
$$

A:

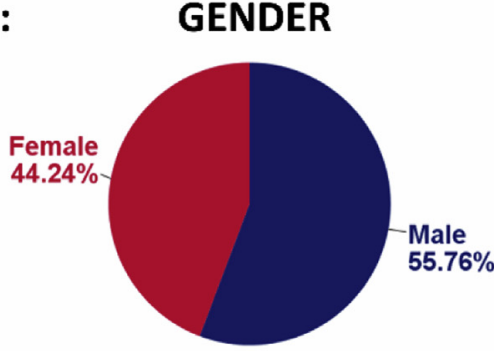

B:

AGE

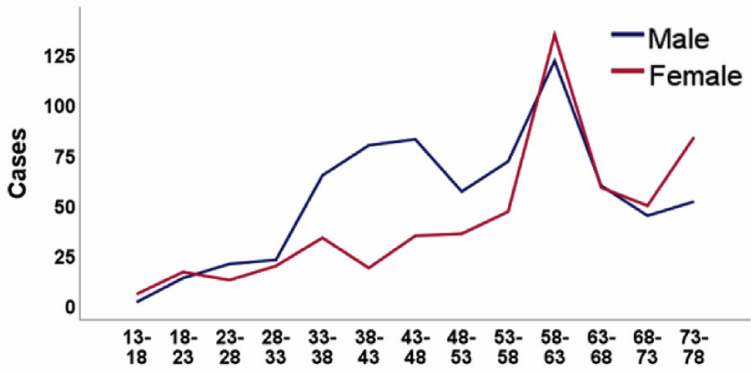

C:

SEVERITY

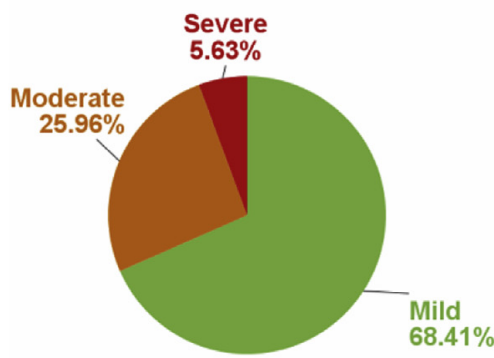

D: MORTALITY

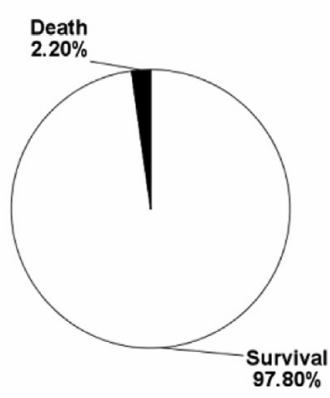

E:

LOH

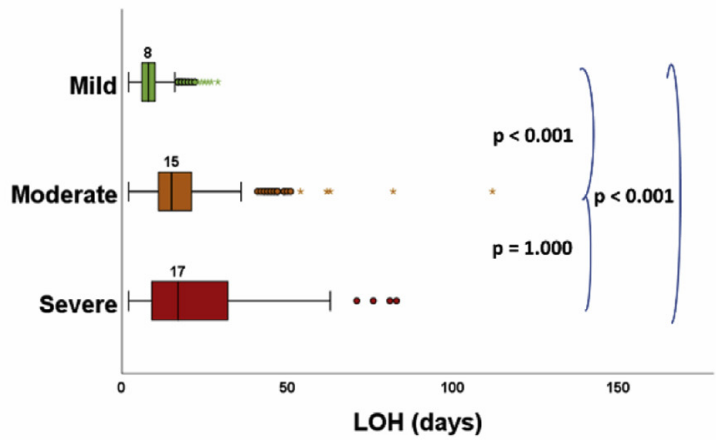

\section{F: DRUG VS. OTHER ETIOLOGIES OF ACUTE PANCREATITIS}

\begin{tabular}{|c|c|c|c|c|c|c|c|c|c|c|}
\hline \multirow[b]{3}{*}{ drug induced (first episodes) } & \multirow{2}{*}{\multicolumn{2}{|c|}{ Frequency }} & \multicolumn{6}{|c|}{ Severity } & \multirow{2}{*}{\multicolumn{2}{|c|}{ Mortality }} \\
\hline & & & \multicolumn{2}{|c|}{ Mild } & \multicolumn{2}{|c|}{ Moderate } & \multicolumn{2}{|c|}{ Severe } & & \\
\hline & \multicolumn{2}{|c|}{1060} & 729 & $68.77 \%$ & 118 & $11.13 \%$ & 213 & $20.09 \%^{a}$ & 90 & $8.49 \%^{\mathrm{a}}$ \\
\hline drug induced (rechallenges) & \multicolumn{2}{|c|}{241} & 144 & $83.24 \%$ & 15 & $8.67 \%$ & 14 & $8.09 \%$ & 5 & $2.07 \%$ \\
\hline TOTAL (drug induced) & \multicolumn{2}{|c|}{1301} & 873 & $70.80 \%$ & 133 & $10.79 \%$ & 227 & $18.41 \%^{b}$ & 95 & $7.30 \%{ }^{b}$ \\
\hline biliary & 471 & $38.45 \%$ & 368 & $78.13 \%$ & 84 & $17.83 \%$ & 19 & $4.03 \% *$ & 5 & $1.06 \%^{* * *}$ \\
\hline idiopathic & 243 & $19.84 \%$ & 157 & $64.61 \%$ & 64 & $26.34 \%$ & 22 & $9.05 \% * *$ & 9 & $3.70 \%^{* *}$ \\
\hline \multirow{2}{*}{ alcoholic } & 184 & $15.02 \%$ & 110 & $59.78 \%$ & 68 & $36.96 \%$ & 6 & $3.26 \%$ & 4 & $2.17 \%{ }^{* *}$ \\
\hline & 44 & $3.59 \%$ & 20 & $45.45 \%$ & 18 & $40.91 \%$ & 6 & $13.64 \%$ & 0 & $0.00 \%{ }^{*}$ \\
\hline post ERCP & 34 & $2.78 \%$ & 24 & $70.59 \%$ & 10 & $29.41 \%$ & 0 & $0.00 \%$ & 0 & $0.00 \%$ \\
\hline others (virus infection, trauma, etc.) & 100 & $8.16 \%$ & 64 & $64.00 \%$ & 30 & $30.00 \%$ & 6 & $6.00 \%$ & 4 & $4.00 \%$ \\
\hline \multirow{2}{*}{\begin{tabular}{|l|} 
alcoholic + biliary \\
alcoholic + lipid metabolic disorder
\end{tabular}} & 23 & $1.88 \%$ & 16 & $69.57 \%$ & 6 & $26.09 \%$ & 1 & $4.35 \%^{*}$ & 2 & $8.70 \%$ \\
\hline & 17 & $1.39 \%$ & 8 & $47.06 \%$ & 6 & $35.29 \%$ & 3 & $17.65 \%^{*}$ & 0 & $0.00 \%$ \\
\hline \multirow{2}{*}{\begin{tabular}{|l|} 
other combination \\
TOTAL (other etiologies)
\end{tabular}} & 109 & $8.90 \%$ & 71 & $65.14 \%$ & 32 & $29.36 \%$ & 6 & $5.50 \%$ & 3 & $2.75 \%{ }^{*}$ \\
\hline & & & 838 & $68.41 \%$ & 318 & $25.96 \%$ & 69 & $5.63 \%$ & 27 & $2.20 \%$ \\
\hline
\end{tabular}

Supplementary Figure 1. The general cohort of AP: $(A)$ sex ratio, $(B)$ age distribution, $(C)$ severity of $A P,(D)$ mortality, and $(E)$ $\mathrm{LOH}$ stay ( $\mathrm{n}=$ number of cases.) The horizontal line in the middle of each box indicates the median; the top and bottom borders of the box mark the 75th and 25th percentiles, respectively; the whiskers mark the minimum and maximum of all of the data; and the circles indicate outliers. $(F)$ Severity and mortality rates of DIAP compared with the other etiologies of $P$. a: druginduced first episodes vs drug-induced rechallenges, $P<.001$; b: all DIAP vs all AP of other etiologies, $P<.001 ;{ }^{* \star \star} P<.001$; ${ }^{\star \star} P<.01 ;{ }^{\star} P<.05$. Further significant differences are marked with stars for other etiology vs first episodes of DIAP. ${ }^{\star \star \star} P<$ $.001,{ }^{\star \star} P<.01,{ }^{\star} P<.05$. 